\title{
An Overview of Carvedilol Side Effects and it's Importance in Medicine and Industry
}

\author{
Omar G. Mousa, Emad Yousif*, Mohammed H. Al-Mashhadani* \\ Department of Chemistry, College of Science, Al-Nahrain University, Baghdad, Iraq
}

*Corresponding Author: Mohammed H. Al-Mashhadani, Department of Chemistry, College of Science, Al-Nahrain University, Baghdad, Iraq

\begin{abstract}
Carvedilol is a medication which is utilized to treat congestive cardiac inability and high blood pressure. It is a nonselective $\beta$-adrenergic blocking agent with vasodilating activity. It used in many field, such as inhibition of carbon steel corrosion and inhibition of lipid Peroxidation.
\end{abstract}

Key words: Carvedilol, Lipid peroxidation, inhibition, high blood pressure.

\section{INTRODUCTION}

\subsection{History of the drug}

Carvedilol is a $\beta$-adrenoreceptor antagonist medication with an $\alpha_{1}$ - adrenoreceptor antagonist effect. It was accepted in September 1995 in the United States for the care of patients with high blood pressure and in May 1997 on the basis of the findings of multiple clinical trials, ${ }^{1-8}$ It was the first adrenoceptorblocking medication to be accepted for symptomatic heart failure treatment.

\section{MATERIALS AND RESUltS}

\subsection{Description}

Carvedilol can be defined as one of the racemic mixtures, in which $\mathrm{S}(-)$ enantiomer is one of the beta adrenoceptor blockers and $\mathrm{R}(+)$ enantiomer is beta as well as alpha-1 adrenoceptor blocker., ${ }^{9,10}$ presently, it is utilized for the treatment of the heart failures, hypertension, and left ventricular dysfunctions. Carvedilol's dual action has been proven to be beneficial in the combination types of therapy as small dosages of two drugs have reduced incidences of the negative consequences in comparison with the high dosage mono-therapy in treating moderate hypertension. ${ }^{11}$

Carvedilol is the generic 1-(9H-carbazol-4-yloxy)-3-[[2-(2-methoxyphenoxy) ethyl]amino]-2propanol as shown in Figure 1.

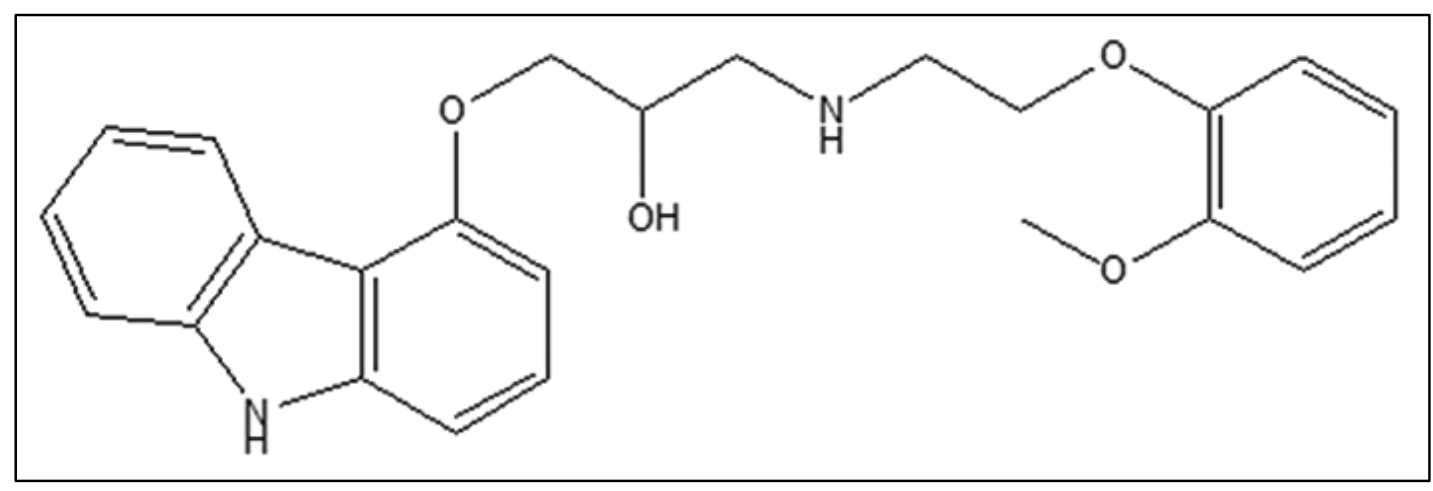

Figure1. Carvedilol chemical structure

Carvedilol has a molecular weight 406.50 with a chemical formula $\mathrm{C}_{24} \mathrm{H}_{26} \mathrm{~N}_{2} \mathrm{O}_{4}$. It is a white powder and easily soluble in dimethylsulfoxide, methanol, and methylene chloride. Hence, carvedilol is moderately soluble in $94 \%$ ethanol and isopropanol; it is partially soluble in ethyl ether; and is almost water insoluble. 


\subsection{Synthesized of Carvedilol}

Carvedilol (10) was synthesized by two steps as shown in Scheme 1. First step was reacting4-hydroxy carbazole (6) with epichlorohydrin(7) using DMSO as a solvent and in the existence of strong base $\mathrm{NaOH}$ to produce 4-oxiranylmethoxy-9H-carbazole (8). In the other step compound 8 reacts with 2(2-methoxy-phenoxy)-ethylamine (9) in the presence of monoglyme to give the target product Carvedilol (10).

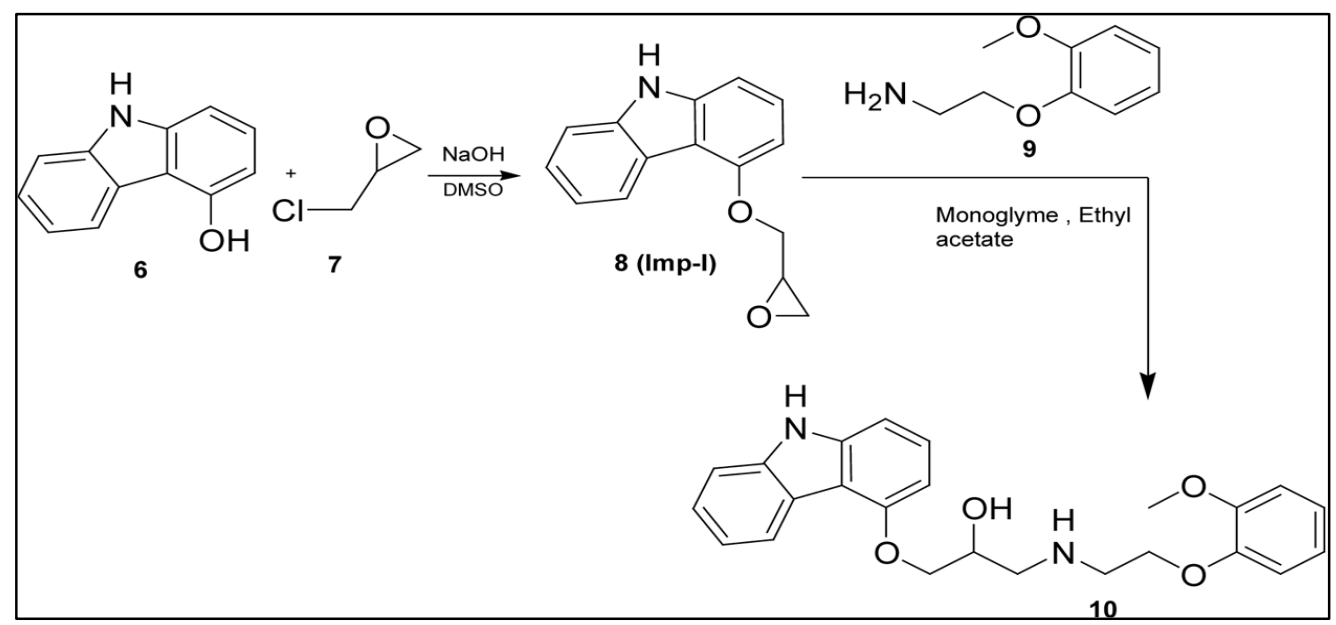

Scheme1. Synthesis route of carvedilol

\subsection{Characterization by Fourier Transform Infrared (FTIR) Spectroscopy}

An IR spectrum of pure carvedilol displayed distinctive peaks at $3344.57 \mathrm{~cm}^{-1}(\mathrm{~N}-\mathrm{H}$ and $\mathrm{O}-\mathrm{H}$ stretching peaks combined together), $3059.10 \mathrm{~cm}^{-1}\left(\mathrm{C}-\mathrm{H}\right.$, stretching , $\left.\mathrm{Sp}^{2}\right), 2924.09 \mathrm{~cm}^{-1}(\mathrm{C}-\mathrm{H}$, stretching, $\mathrm{Sp}^{3}$ ), $1589.34 \mathrm{~cm}^{-1}$ (N-H bending vibrations), $1253.73 \mathrm{~cm}^{-1}$ due to C-O group (epoxides), $1099.43 \mathrm{~cm}^{-1}$ due to aryl alkyl ethers and alkyl ether C-O stretching respectively and $1022.27 \mathrm{~cm}^{-1}$ (symmetric C-O-C stretching) (Figure 2). ${ }^{12-14}$

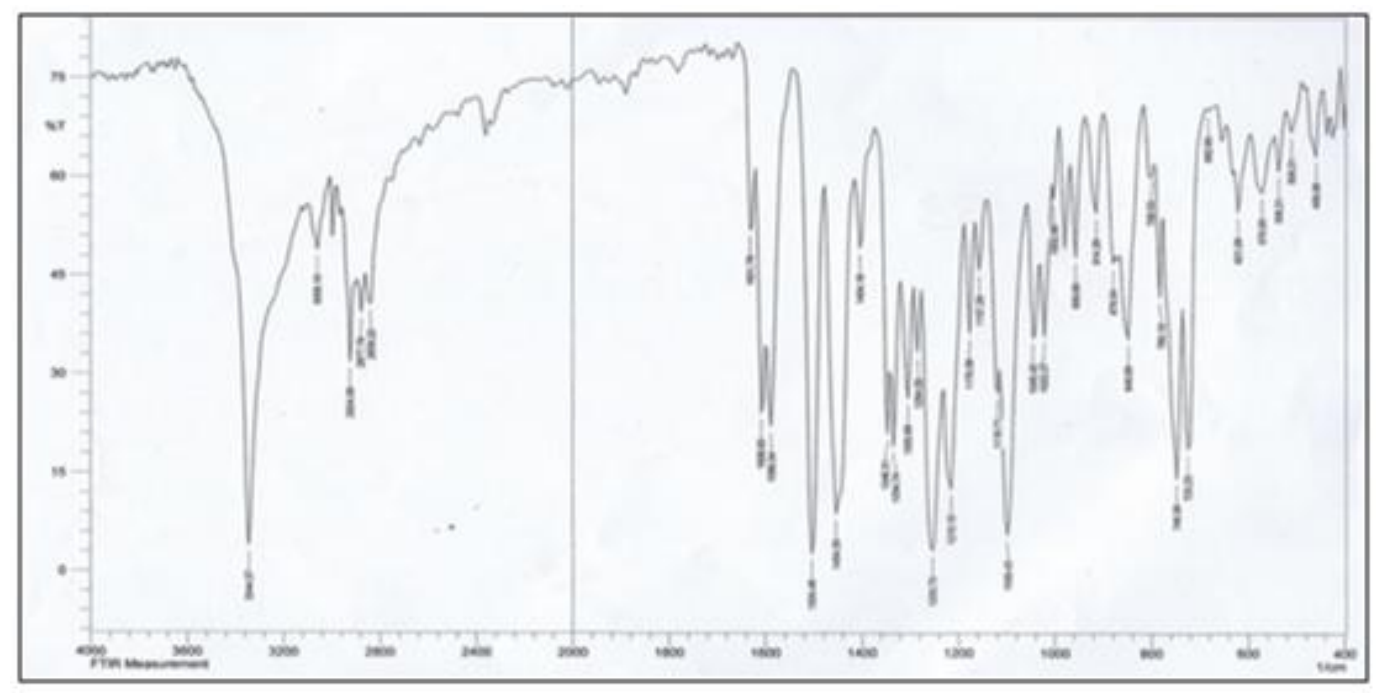

Figure2. FTIR spectrum of Carvedilol

\subsection{Clinical Effectiveness}

- Congestive cardiac inability

- High blood pressure (HBP)

- Coronary heart disease (CHD)

\subsection{Carvedilol Side Effects}

Carvedilol oral tablet can cause several types of side effects, including drowsiness. Don't drive, use machinery, or perform activities that require alertness until you know how this drug affects you. 
More common side effects

The more common side effects that can occur with carvedilol oral tablet include:

- Vertigo

- Unusual lassitude

- low blood pressure

- Diarrhoea

- high blood sugar

- Energy shortage or weakness

- decreased heart rate

- Gaining weight

- changes in sex drive or performance

- dry eyes

- dry, itchy skin

\section{DISCUSSION}

\subsection{Expired Carvedilol Drug used as Inhibition of Carbon Steel Corrosion}

Carbon steel (CS) can be considered as the most commonly utilized material all over the world for theindustrial as well asthe domestic applications, owing to its excellentmechanical characteristics, the fact that it is available, in addition to its fairly low prices. The Hydrochloric acid( $\mathrm{HCl})$ has been commonly utilized in numeroustechnological procedures in the industry (in extracting and processing gas and oil, pickling ponds, and so on) and in many petrochemical and chemical sectors. The carbon steel corrosion behaviors in acidic media is greatly interesting because of its significance in the applications. One of the strongest ways of preventing alloy steel from corrosion has been found to include utilizing synthetic organic compounds as deactivator of corrosion ${ }^{15-17}$ Which are chemical compounds decreases a material's corrosion rate ${ }^{18,19}$. Nevertheless, many of these substances are sadly harmful to both humans and the climate. ${ }^{20}$ Because of the toxic effects of certain corrosion inhibitors, study is aimed on introducing new, safe, nontoxic and environmentally friendly natural products like the compounds of pharmaceutics, leaves, fruit or seed extract that may be utilized as green Inhibitors for Protection of Metals.Numerous studies have recently been conducted on drug use as possible candidates for metal corrosion inhibitions via their functional groups.The organic inhibitors include heteroatomes in common. Nitrogen, sulfur, and Oxygenhave been observed to be having higher electron density and basicity which is why they serve as an inhibitor of corrosion. Nitrogen, sulfur, and Oxygen are important centers for the adsorption process on the metal surface. Antibiotics come under the group of eco-friendly compounds and are thus known to be green correction inhibitors that are fully water soluble and obtainable with a high level of the purity and fairly inexpensive. Those characteristics would be validating their utilization as inhibitors of the corrosion in different mediums.

Similarly, using expired medications as nontoxic inhibitors of the corrosion for the carbon steel in the acidic mediums has been stated. ${ }^{21-25}$ The use of ineffective medicines (expired) as inhibitors of corrosion may betracked back to 2009\&2011, when Abdul-Hamid utilized the obsolete ranitidine as a carbon steel corrosion inhibitor ${ }^{22}$ and for slight steel ${ }^{22}$ in the corrosive medium of the hydrochloric acid, respectively. Ineffective drugs (expired) such as carvedilol are sufficiently large (formula $=\mathrm{C}_{24}$ $\mathrm{H}_{26} \mathrm{~N}_{2} \mathrm{O}_{4}$; molecular weight $=406.50$ ) and are possible to easily cover more carbon steel surfaces (as a result of the adsorption). In comparison, medication Carvedilol is highly inexpensive, environmentally safe,readily available, and nontoxic. Because of these features, the medication Carvedilol was selected for corrosion studies.

An expired Carvedilol, has beenresearchedwith the use of thepotentiodynamic polarization, weight loss, electro-chemical frequency modulation (EFM), and electro-chemical impedance spectroscopy (EIS) approaches as a safe drug for the prevention of theCS corrosions in the acidic media. The 
suppression capacity improved with increased dosage of drugs and has been decreased with higher temp., up to the point where it reaches a maximal $98.94 \%$ value at $25^{\circ} \mathrm{C}$ and $1.60 \times 10^{-4} \mathrm{M}$.Carvedilol prevention of carbon steel corrosion can be a result of the tendency of drug molecules to be adsorbed on metal surface reactive sites. The procedure of the adsorption is following the isotherm of Langmuir through the physical adsorptions. CS surface morphologieshave beenobserved by the AFM. Results which have been obtained from variousapproachesconsiderably coincide. Figure 3 illustrates spectrum of EFM which has been obtained for the Carbon Steel corrosions in $1 \mathrm{M}$ of the solution of the Hydrochloric acid with the presence and absence of $1 \times 10^{-4} \mathrm{M}$ of researched Carvedilol at a temperature of $25^{\circ} \mathrm{C}$, respectively.

(a)

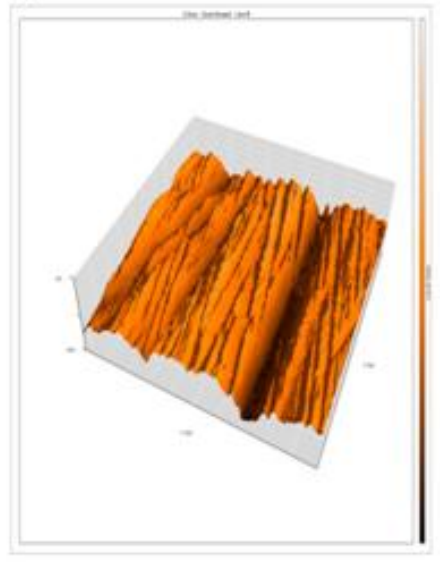

(b)

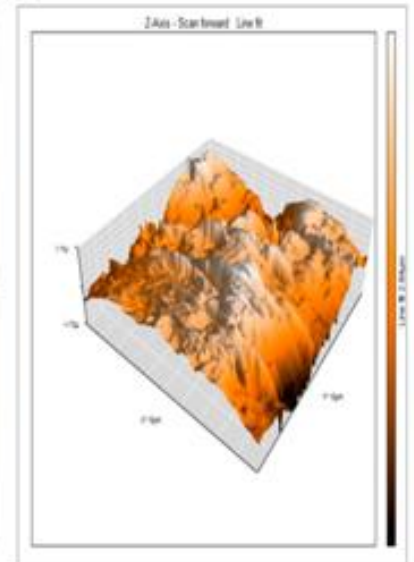

(c)

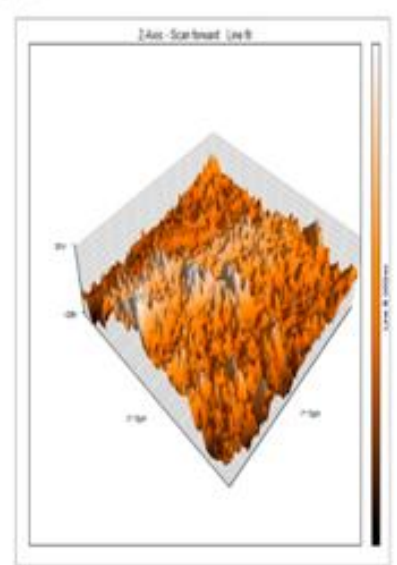

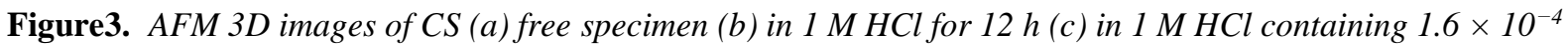
$M$ for 12 hexpired Carvedilol drug at $25^{\circ} \mathrm{C}$

\subsection{Lipid Peroxidation Carvedilol Inhibition. ${ }^{26}$}

Carvedilol prevents the sonicated phosphatidylcholine liposome peroxidationhas been triggered with adding $\mathrm{FeC}_{2}$ while thepindolol, labetalol, and atenolol, have been ineffective. Inhibition has proven not being ascribable (i) to impact the autoxidation of the $\mathrm{Fe}$ (II) and as a result on generating radical initiators that are oxygen derived; (i) to inorganic initiators $\mathrm{O}^{-}$and $\mathrm{OH}$ scavenging (iii) to impact the organic hydro-peroxides reductive cleavage with the $\mathrm{FeCl}_{2}$; (iv) to organic initiators' scavenging. Observations of the fact that (i) carvedilol's efficacyhas been inversely proportionate with $\mathrm{FeCI}_{2}$ and lipid hydro-peroxides' concentration in test.; (ii) the medication results in the prevention of lipid peroxidation onset which is triggeredwith adding $\mathrm{FeCl}_{3}$; (iii) it could result in forming $\mathrm{Fe}$ (III) complex and suggesting a carvedilol action molecular mechanism. It is capable of inhibiting the lipid peroxidation through the attachment ofFe(III), which is produced through the lipid hydro-peroxides in substrate throughout Fe(II) oxidation. The lag time which isintroduce by the carvedilol in peroxidative procedure would be corresponding to time which is taken for the carvedilol to be titrated with the $\mathrm{Fe}$ (III); in the case of the drug consumed Fe(III) accumulates for reaching critical parameter which results in the stimulation of the peroxidation. Based onsuch molecular mechanisms, the anti-oxidant potency of the carvedilol mayresult in its capability for binding a species, $\mathrm{Fe}(\mathrm{III})$, which is a catalyst of the process and to the lipophilic nature, concentrating it in membranes, in which the Fe(III) is produced by a mechanism which is site-specific.

\subsection{Cardiac Mitochondrial Drug-Induced (Toxicity And Protection). ${ }^{27}$}

In addition to its role in the production of energy, Mitochondria has long been involved in many biological processes. The significance of such organelle for the homeostasis of the cardiac tissueswashighly researched and its impairmentsmayresult in the death of the cells and the subsequent failure of organ. Numerous compounds werediscussed in literature as having direct impacts on the cardiac mitochondria that is capable of providing mechanistic explanationsof their pharmacological or toxicological impacts. The presentedevaluationis a description of one of the common examples of the drug-induced cardiac mitochondrial toxicitiesas well as another drug-induced mitochondrial protection case. For the first, the doxorubicin case has been presented, an anti-cancer agent in which the treatment is related to a dose-dependent and cumulative cardio-myopathywith mitochondrial etiology. After that, carvedilol case has been presented, a -blocker with the inherent anti-oxidant 
activitiesthat have been described for protecting the cardiac mitochondria from the oxidative injuries. The last part of this review includes an integration of the information from preceding chapters, which demonstrate the way that the carvedilol is capable of contributing to the reduction of the toxicity of the doxorubicin on the cardiac mitochondria. Both examples that have been discussed resulted in significant key messages: i) the drug-induced cardiac mitochondrial dysfunction is one of thesignificant contributors for the drug-related failures of organs, ii) protecting the mitochondrial functionshas beenincluded in the usefuleffect of a few of theclinically-utilizedmedicarions, iii) a more precisetoxic versususefulimpact predictionshas to be one of thesignificant drug development components by the industry of the pharmaceutics.

\subsection{Metal-Chelating Features of Carvedilol. ${ }^{28}$}

Carvedilol, that is considered as one of the anti-hypertensive agents with the $\beta$-blocker function as well as anti-oxidant activities might be specified as a drug with chelating features, thus it might be applied in chelation therapy. With regard to the general context related to potential activity in biomedical chemistry, it was indicated that a few drug's coordination by metal ions enhanced the drug's pharmaceutical activity and decreased their un-wanted impacts in human as well as veterinary medicine. In the case of this work, the carvedilol coordination to $\mathrm{Fe}(\mathrm{III}), \mathrm{Zn}$ (II), in addition to $\mathrm{Cu}$ (II) might be considered in the capability of a few drugs in sequestering excess of the metal ions and therefore exerting their anti-oxidant activity. The results of this work in sequestering $\mathrm{Zn}$ (II), Fe (III) and already-indicated $\mathrm{Cu}$ (II) capability regarding carvedilol can be shown in cellular model, there is a possibility that the drug might be utilized for therapy of diseases which are characterized via $\mathrm{Cu}$ (II), $\mathrm{Zn}$ (II), and Fe (III) accumulation. In addition, the metal ions participate in generating oxygen free radicals via Fenton as well as Weiber-Weiss reactions. Therefore, the capability of carvedilol in sequestering the metal ions might be inducing its anti-oxidative effect.

\section{CONCLUSiON}

Carvedilol is a very known therapy which can be utilized for congestive heart failure, hypertension and ischemic heart disease. It also used in many. It used in many field, such as inhibition of carbon steel corrosion and Inhibition of Lipid Peroxidation.

\section{REFERENCES}

[1] Metra M, Nardi M, Giubbini R, Dei Cas L. Effects of short- and long-term carvedilol administration on rest and exercise hemodynamic variables, exercise capacity and clinical conditions in patients with idiopathic dilated cardiomyopathy. J Am CollCardiol 1994;24:1678-1687

[2] Krum H, Sackner-Bernstein JD, Goldsmith RL, et al. Double-blind, placebo-controlled study of the longterm efficacy of carvedilol in patients with severe chronic heart failure. Circulation1995;92:1499-150

[3] Olsen SL, Gilbert EM, Renlund DG, Taylor DO, Yanowitz FD, Bristow MR. Carvedilol improves left ventricular function and symptoms in chronic heart failure: a double-blind randomized study. J Am CollCardiol 1995;25:1225-1231

[4] Bristow MR, Gilbert EM, Abraham WT, et al. Carvedilol produces dose-related improvements in left ventricular function and survival in subjects with chronic heart failure. Circulation 1996;94:2807-2816

[5] Packer M, Colucci WS, Sackner-Bernstein JD, et al. Double-blind, placebo-controlled study of the effects of carvedilol in patients with moderate to severe heart failure: the PRECISE trial: Prospective Randomized Evaluation of Carvedilol on Symptoms and Exercise. Circulation 1996;94:2793-2799

[6] Colucci WS, Packer M, Bristow MR, et al. Carvedilol inhibits clinical progression in patients with mild symptoms of heart failure. Circulation 1996;94:2800-2806.

[7] Cohn JN, Fowler MB, Bristow MR, et al. Safety and efficacy of carvedilol in severe heart failure. J Card Fail 1997;3:173-179

[8] Australia/New Zealand Heart Failure Research Collaborative Group. Randomised, placebo-controlled trial of carvedilol in patients with congestive heart failure due to ischaemic heart disease. Lancet1997;349:375380

[9] Hokama N, Hobara N, Sakai M, Kameya H, Ohshiro S, Sakanashi M: Influence of nicardipine and nifedipine on plasma carvedilol disposition after oral administration in rats. J Pharm Pharmacol. 2002 Jun;54(6):821-5

[10] Kakumoto M, Sakaeda T, Takara K, Nakamura T, Kita T, Yagami T, Kobayashi H, Okamura N, Okumura K: Effects of carvedilol on MDR1-mediated multidrug resistance: comparison with verapamil. Cancer Sci. 2003 Jan;94(1):81-6 
[11] Jonsson O, Behnam-Motlagh P, Persson M, Henriksson R, Grankvist K: Increase in doxorubicin cytotoxicity by carvedilol inhibition of P-glycoprotein activity. BiochemPharmacol. 1999 Dec 1;58(11):1801-6

[12] Krstić M, Radojević M, Stojanović D, Radojević V, Uskoković P, Ibrić S. Formulation and characterization of nanofibers and films with carvedilol prepared by electrospinning and solution casting method. Eur J Pharm Sci 2017; 101:160-166.

[13] Nagy ZK, Balogh A, Drávavölgyi G, Ferguson J, Pataki H, Vajna B, et al. Solvent-free melt electrospinning for preparation of fast dissolving drug delivery system and comparison with solvent-based electrospun and melt extruded systems. J Pharm Sci 2013; 102:508-517.

[14] Saini R, Singh SK, Verma PRP. Evaluation of carvedilolloadedmicrosponges with nanometricpores using response surface methodology. J ExpNanosci 2014; 9:831-850.

[15] Ahmad, I.; Prasad, R.; Quraishi, M.A. Thermodynamic, Electrochemical and Quantum Chemical Investigation of Some Schiff Bases as Corrosion Inhibitors for Mild Steel in Hydrochloric Acid Solutions. Corros. Sci. 2010, 52, 933-942.

[16] Yurt, A.; Aykin, O. Thermodynamic, Electrochemical and Quantum Chemical Investigation of Some Schiff Bases as Corrosion Inhibitors for Mild Steel in Hydrochloric Acid Solutions. Corros. Sci. 2011, 53, 3725-3732.

[17] Chetouani, A.; Hammouti, B.; Benhadda, T.; Daoudi, M. Inhibitive Action of Bipyrazolic Type Organic Compounds Towards Corrosion of Pure Iron in Acidic Media. Appl. Surf. Sci. 2005, 249, 375-385.

[18] Quraishi, M.A.; Sardar, R. Aromatic Triazoles as Corrosion Inhibitors for Mild Steel in Acidic Environments. Corrosion 2002, 58, 748-755.

[19] Juttner, K. Electrochemical Impedance Spectroscopy (EIS) of Corrosion Processes on Inhomogeneous Surfaces. Electrochim.Acta. 1990, 35, 1501-5108.

[20] Ostovari, A.; Hoseinieh, S.M.; Peikari, M.; Shadizadeh, S.R.; Hashemi, S.J. Corrosion Inhibition of Mild Steel in $1 \mathrm{M} \mathrm{HCl}$ Solution by Henna Extract: A Comparative Study of the Inhibition by Henna and its Constituents (Lawsone, Gallic Acid, $\alpha$-d-Glucose and Tannic Acid). Corros. Sci. 2009, 51, 1935-1949.

[21] Abdel Hameed, R.S. Expired Ranitidine Drugs as Corrosion Inhibitor for Aluminum in $1 \mathrm{M} \mathrm{HCl}, \mathrm{Al}$ Azhar. Bull. Sci. 2009, 20, 151-163.

[22] Abdel Hameed, R.S. Ranitidine Drugs as Non-Toxic Corrosion Inhibitors for Mild Steel in HCl Medium, Port. Electrochim.Acta. 2011, 29, 273-285.

[23] Al-Shafey, H.I.; Abdel Hameed, R.S.; Ali, F.A.; Aboul-Magd, A.S.; Salah, M. Effect of Expired Drugs as Corrosion Inhibitors for Carbon Steel in 1M HCL Solution. Int. J. Pharm. Sci. Rev. Res. 2014, 27, 146152.

[24] Abdel Hameed, R.S. Review Expired Drugs as Corrosion Inhibitors for Metals and Alloys. Phys. Chem. Ind. J. 2013, 8 (4), 146-149.

[25] Fouda, A.S.; Mahmoud, W.M.; Abdul Mageed, H.A. Evaluation of an Expired Nontoxic Amlodipine Besylate Drug as a Corrosion Inhibitor for Low-CS in Hydrochloric Acid Solutions. J. Bio. TriboCorros. 2016, 2 (7), 1-11.

[26] TADOLINI, Bruna; FRANCONI, Flavia. Carvedilol inhibition of lipid peroxidation.A new antioxidative mechanism. Free radical research, 1998, 29.5: 377-387.

[27] Paulo J. Oliveira, Drug-induced Cardiac Mitochondrial Toxicity and Protection: From Doxorubicin to Carvedilol. Current Pharmaceutical Design, 2011, 17, 2113-2129.

[28] Zoroddu, Maria Antonietta, Serenella Medici, and Massimiliano Peana. "Metal-chelating properties of carvedilol: an antihypertensive drug with antioxidant activity." Journal of Coordination Chemistry 62.23 (2009): 3828-3836.

Citation: Mohammed H. Al-Mashhadani, et.al, "An Overview of Carvedilol Side Effects and it's Importance in Medicine and Industry”, International Journal of Advanced Research in Chemical Science, 7(4), pp. 36-41. DOI: https:// doi.org/10.20431/2349-0403.0704004

Copyright: (C) 2020 Authors, This is an open-access article distributed under the terms of the Creative Commons Attribution License, which permits unrestricted use, distribution, and reproduction in any medium, provided the original author and source are credited. 